\title{
The neural basis for response latency in a sensory-motor behavior
}

\section{Joonyeol Lee ${ }^{1,2}$, Timothy R. Darlington ${ }^{3}$, and Stephen G. Lisberger ${ }^{3}$}

${ }^{1}$ Center for Neuroscience Imaging Research, Institute for Basic Science (IBS), Suwon 16419, Republic of Korea

${ }^{2}$ Department of Biomedical Engineering, Sungkyunkwan University, Suwon 16419, Republic of Korea

${ }^{3}$ Department of Neurobiology, Duke University School of Medicine, Durham, NC, USA

Running Title: Neural basis of sensory-motor latency

Word count: Abstract, 196; Introduction, 724; Discussion, 1373

Figures: Total, 9; Color, 7

Acknowledgments: We thank S. Tokiyama, S. Ruffner, and S. Happel for technical assistance. We also thank Dr. J. Patrick Mayo for help in visual stimulus timing calibration. Research supported by NIH grant R01-EY027373 (SGL) and F30-EY027684 (TRD). Joonyeol Lee was partly supported by IBS-R015-D1.

The authors declare no competing financial interests.

Keywords: movement latency, smooth pursuit eye movements, correlated variation, neuronbehavior correlations, neuron-neuron correlation, frontal eye fields, local field potential

Author contributions: Conceptualization, J.L and S.G.L.; Methodology, J.L. and S.G.L.; Investigation, J.L. and TRD; Formal Analysis, J.L. and TRD; Writing - Original Draft, J.L. and S.G.L.; Writing - Review and Editing, J.L., T.R.D, and S.G.L.; Funding Acquisition, S.G.L.

Proofs and correspondence to:

Stephen G. Lisberger

Department of Neurobiology

Duke University School of Medicine

311 Research Drive, Room 101

Durham, NC 27710

Email: lisberger@neuro.duke.edu 


\begin{abstract}
We seek a neural circuit explanation for sensory-motor reaction times. We have found evidence that two of three possible mechanisms could contribute to reaction times in smooth pursuit eye movements. In the smooth eye movement region of the frontal eye fields (FEFSEM), an area that causally affects the initiation of smooth pursuit eye movement, neural and behavioral latencies have significant trial-by-trial correlations that can account for $40 \%$ to $100 \%$ of the variation in behavioral latency. The amplitude of preparatory activity, which represents the motor system's expectations for target motion, shows negative trial-by-trial correlations with behavioral latency and could contribute to the neural computation of reaction time. In contrast, the traditional "rampto-threshold" model is contradicted by the responses of many, but not all FEF SEM neurons. As evidence of neural processing that determines reaction time, the local field potential in FEFSEM includes a brief wave in the $5-15 \mathrm{~Hz}$ frequency range that precedes pursuit initiation and whose phase is correlated with the latency of pursuit in individual trials. We suggest that the latency of the incoming visual motion signals combines with the state of preparatory activity to determine the latency of the transient response that drives eye movement.
\end{abstract}




\section{Introduction}

When a major league baseball hitter faces a 95-mile-per-hour fastball, he has just over $400 \mathrm{~ms}$ from when the pitcher releases the ball to the time of contact with his bat. Extensive neural processing must occur in this short time to (1) process the visual inputs from the baseball, (2) decide whether or not to swing the bat, and (3) initiate a movement that is accurate in both location and timing. Timing is particularly important because a difference of a few milliseconds in the timing of the swing will determine whether or not the bat contacts the ball and whether the ball is driven into fair or foul territory. The temporal precision and accuracy of highly-skilled movements focuses attention on the neural mechanisms that decide exactly when to initiate a movement.

In the present paper, we ask what features of neural responses control behavioral latency using visually-guided smooth pursuit eye movements as a model system. Pursuit is driven by visual motion with latencies just under $100 \mathrm{~ms}$, and is initiated in the rich form we study only in the presence of visual motion. The visual inputs that drive pursuit arise from extrastriate area MT (Newsome et al., 1988), where latencies to visual motion average around $60 \mathrm{~ms}$ (Lisberger and Movshon, 1999). Thus, only 40 milliseconds are available to transform visual motion signals into movement. We tend to think of the latency of pursuit in terms of the times required for visual signals to arrive in MT, traverse the cortex to the smooth eye movement region of the frontal eye field $\left(\mathrm{FEF}_{\mathrm{SEM}}\right)$, reach neurons in the pontine nuclei and the nucleus reticularis tegmenti pontis, and drive the simple-spike activity of Purkinje cells in the floccular complex of the cerebellum.

From first principles, however, multiple features of neural responses might control behavioral latency. One popular model posits that neural activity at a key site ramps up to a threshold that triggers movement. Faster ramps will reach threshold more quickly and trigger a movement with a shorter latency. This model appears to hold for saccadic eye movements (Hanes and Schall, 1996), and may apply for decision making, where one theory posits that ramping activity during the acquisition of sensory data accumulates evidence and leads to a movement to report a decision when the ramp crosses a threshold (Gold and Shadlen, 2007). However, ramp-to threshold may not apply for all kinds of movements. For example, as we found in area MT, the latency of the visually-driven transient response might be the key feature of neural responses that determines behavioral latency.

FEF $_{\text {SEM }}$ is known from stimulation, lesion, recording, and anatomical studies to be causally involved in the generation of pursuit eye movements (Brodal, 1980; Gottlieb et al., 1994, 1993; Keating, 1991; Lisberger, 2010; MacAvoy et al., 1991; Ono and Mustari, 2009; Shi et al., 1998; Tanaka and Fukushima, 1998; Tanaka and Lisberger, 2001, 2002b, 2002a). It is a combined sensory and motor area and plays a role in setting the strength of the pursuit response based on previous experience and the signal-to-noise ratio of the sensory signal. The responses of neurons in FEF $_{\text {SEM }}$ are rich enough to allow tests of multiple neural correlates of pursuit latency. The responses include both transient pulses of firing related to the initiation of pursuit and preparatory activity that evolves before a moving target initiates pursuit. Thus, we can test the ramp-tothreshold theory as well as the potential roles of the amplitude of preparatory activity versus the latency of the pursuit-related response in determining behavioral latency.

We find evidence for multiple computations that may control pursuit latency, with at best weak evidence in favor of the ramp-to-threshold theory. The trial-by-trial variation in the latency of 


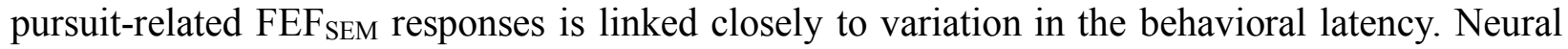
latency can account for 40 to $100 \%$ of the variation in behavioral latency. The amplitude of preparatory activity is negatively correlated with pursuit latency in many neurons, and could contribute to the latency computation. We also find a low frequency component in the local-field potential (LFP) that, itself, shifts in time (or phase) in relation to pursuit latency. We suspect that both the latency of the incoming visual motion signals and the expectation represented by preparatory activity contribution to neural and behavioral latency, and that the low-frequency LFP holds a key to understanding how they combine.

\section{Results}

Our goal is to understand the neural circuit mechanisms that determine sensory-motor latency. Our analysis focuses on the trial-by-trial variation between neural and behavioral responses because trial-by-trial correlations provide a powerful tool for understanding the processing that occurs within a sensory-motor system. For example, our previous paper (Lee et al., 2016) implies that variation in the latency of responses in area MT could account for up to $70 \%$ of the trial-by-trial variation in the latency of pursuit.

Our strategy was to record local field potentials (LFP) and the spiking activity of multiple single neurons in the smooth eye movement region of the frontal eye fields (FEFSEM) while monkeys pursued a spot target that underwent step-ramp motion (Figure 1A, after Rashbass, 1961). Because we were interested in evaluating single-trial data and revealing trial-by-trial correlations between neural and behavioral responses, we typically collected more than 100 repetitions of a few target motions (Figure 1B).

Here, we present new results on the features of responses in FEFSEM that contribute to neural and behavioral latency, and we compare our results in FEFSEM with additional analyses of the data from prior recordings in area MT (Lee et al., 2016). We report three main findings. (1) The local field

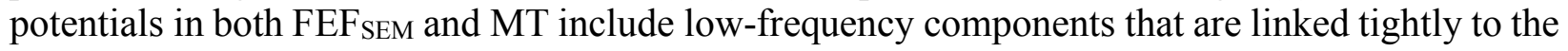
latency of smooth pursuit eye movements and that are a signature of trial-by-trial correlations between the latencies of neighboring neurons. (2) The trial-by-trial variation in the latency of spiking responses in FEF SEM $_{\text {is }}$ tightly correlated with the latency of pursuit eye movements, with the expected commensurate neuron-neuron latency correlations for pairs of FEF (Schoppik et al., 2008). (3) The amplitude of preparatory activity is correlated negatively with

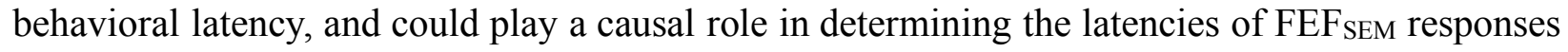
and pursuit eye movement.

\section{Trial-by-trial correlation between neural latency and pursuit latency}

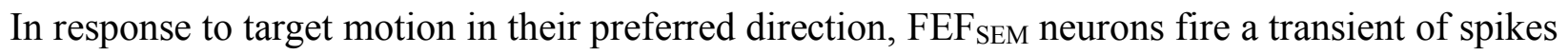
followed by a sustained plateau, frequently at a lower rate (Figure 1B). As illustrated in Figure 1B, the onset of FEFSEM responses usually precedes the onset of pursuit eye velocity, and both the firing rate response and the eye velocity of pursuit vary considerably in both latency and amplitude from trial-to-trial. Here, we leverage those variations to discover features of the neural computations that determine sensory-motor latency in the pursuit system. 
Trial-by-trial correlations between the latency variations of FEFSEM neurons and pursuit eye movements provide evidence that $\mathrm{FEF}_{\mathrm{SEM}}$ controls sensory-motor reaction time to some degree. We used a procedure described elsewhere to estimate trial-by-trial latencies for pursuit eye movements (Lee et al., 2016; Lee and Lisberger, 2013) and to sort behavioral trials into 5 quintiles based on the estimated latency of pursuit in each trial (Figure 2A). We then revealed coordinated shifts in the latencies of eye movements and neural responses in FEFSEM by averaging the timevarying firing rates in each quintile (Figure 2B).

To quantify the trial-by-trial latency correlations between FEF neuron, we applied the estimation method detailed in our previous publication (Lee et al., 2016). Briefly, we used an iterative Bayesian method to estimate the latency and gain of firing rate and eye velocity on each individual trial relative to a template created by averaging across groups of trials. We made these estimates on the basis of the trajectory of firing rate from -20 to $100 \mathrm{~ms}$ from the onset of neural response and of eye velocity from -20 to $80 \mathrm{~ms}$ after the initiation of smooth pursuit eye movement. By basing our estimates on the full transition of each variable from baseline to peak value, we ensure that our estimates of latency are based on the left-right shift of the full waveform relative to the average, and the estimate of response amplitude is based on the full transition from baseline to peak. We then obtained the Pearson's correlation coefficient between the latencies of firing rate and eye movement by taking advantage of the fact that the correlation coefficient is defined by the regression slope of Z-transformed data (Figure 2C).

The correlation between neural and behavioral latency (Figure 2D) formed a distribution across all our neurons with a mean of 0.19 that was significantly different from zero (t-test $\mathrm{p}=3.4 \times 10^{-22}$, $\mathrm{n}=99$ neurons). The mean correlation coefficients were 0.196 and 0.183 in the two monkeys individually and were significantly different from zero (t-test $\mathrm{p}=7.13 \times 10^{-12}$ and $1.46 \times 10^{-11}, \mathrm{n}=50$ and 49 neurons). In contrast, for the same sample of neurons, the correlation between the amplitude of the pursuit-related responses and behavioral latency had means of 0.007 and -0.010 in the two monkeys and were not significantly different from zero (t-test $\mathrm{p}=0.61$ and 0.61$)$.

The absolute response latencies of FEFSEM neurons $(n=125)$ fit well within the expectations for a sensory-motor circuit. They preceded the initiation of pursuit by an average of $27 \mathrm{~ms}$ (Figure 2E) and followed the responses of MT neurons $(n=135)$ by an average of $13.3 \mathrm{~ms}$ (Figure $2 \mathrm{~F}$ ). The latency of responses in area MT neurons was $40.3 \mathrm{~ms}$ and the distributions of response latency for MT and $\mathrm{FEF}_{\text {SEM }}$ were statistically different (t-test, $\left.\mathrm{p}=4.2 \times 10^{-10}\right)$.

To verify that the small magnitude of neuron-behavior latency correlations was not an artifact of the vagaries of estimating single-trial neural latency from a handful of spikes, we evaluated the relationship between neural response amplitude and neuron-behavior latency correlation. Our premise was that artifacts of latency estimation would mitigate against large correlations in neurons with weak responses. However, we found that neurons with weak responses during pursuit could have either strong or weak neuron-behavior latency correlations while neurons with stronger responses had mainly strong neuron-behavior latency correlations (Figure 2G). Thus, we believe the values of neuron-behavior latency correlation. 


\section{Local field potential in the 5-15 Hz range is linked to pursuit latency}

Local field potential (LFP) provides an index of the neural processing within the localized region around the tip of the recording electrode. We reasoned that analysis of the LFP in our recordings should reveal correlates to confirm the phenomena we measured in spikes, and that the LFP might reveal special features of the neural computation that determines sensory-motor reaction time.

In FEF $_{\text {SEM, }}$, we find a fairly large trial-by-trial correlation between the phase of the LFP and the pursuit latency (Figure 3A). As outlined in the Methods, we applied the Hilbert transform to LFPs that had been filtered with narrow pass bands at different frequencies and obtained instantaneous phase and power for each frequency and every millisecond in every trial. Then, we calculated the trial-by-trial correlation between pursuit latency and the instantaneous phase and power as a function of time. For FEF $_{\text {SEM, }}$, the correlation peaks for frequencies in the 5-15 Hz range and times around $100 \mathrm{~ms}$ after the onset of target motion, near the time of the initiation of pursuit. Analysis of LFP data in area MT (Figure 3B) revealed a similar correlation, albeit with a slightly smaller value of peak correlation in MT for the examples in Figures $3 \mathrm{~A}$ and B.

The finding of a correlation between LFP phase and pursuit latency stimulated us to identify the component of the LFP that is linked to pursuit initiation. We band-pass filtered the raw LFP from each behavioral trial to extract the $5-15 \mathrm{~Hz}$ frequency component. When synchronized on the onset of target motion, the LFP in FEF $\mathrm{SEM}$ from single trials contained a brief wave that started about 30 $\mathrm{ms}$ after the onset of target motion and underwent a negative-positive-negative fluctuation that ended about $200 \mathrm{~ms}$ after the onset of target motion. The shape of the LFP wave was consistent across the 123 LFP recordings as is shown in Figure 3C. In MT, the same analysis yielded a somewhat smaller and more complicated set of deflections, but otherwise the results in the two areas were remarkably similar (138 LFP recordings, Figure 3D). We next asked whether the LFP shifted in time in relation to pursuit latency by grouping the filtered LFP according to the quintiles of pursuit latency for each neuron. For two example neurons, this analysis revealed a relationship

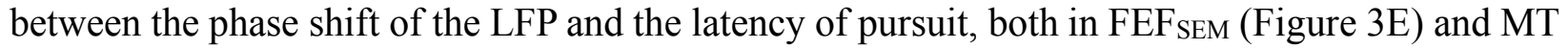
(Figure 3F), consistent with the trial-by-trial correlations shown in Figures 3A and B.

Quantitative analysis of all recording sites from FEFSEM and MT revealed that behavioral latency is much more strongly correlated with the phase shift of the LFP than with its power. Now, we correlated the behavioral latency on each trial with the power and phase in the LFP after filtering with a 5-15 Hz frequency window and plotted the results as a function of the time during the LFP. For an example LFP recorded in FEFSEM (Figure 4A), the correlation between latency and LFP phase reaches a maximum correlation of $\sim 0.6$, while the correlation between latency and LFP power is smaller and negative. Population averages across 123 LFPs in FEF effects (Figure 4C). In the population average, the correlation reaches a peak of 0.25 just more than $100 \mathrm{~ms}$ after the onset of target motion, which corresponds to the time of initiation of smooth pursuit. The results are similar for the LFP in area MT, although the effects are smaller (Figure 4B, D). We conclude that LFP in the $5-15 \mathrm{~Hz}$ frequency range is tightly linked to the latency of pursuit and may be related to neural processing in both MT and FEF motor reaction time. The differences in the details of the LFP wave in MT and FEFSEM and its systematic relationship with behavioral latency strongly imply that the LFPs we found in the two areas are not simply an artifact of the procedures we used for filtering the LFP. 


\section{Relationships among LFP, neuron-behavior, and neuron-neuron latency correlations}

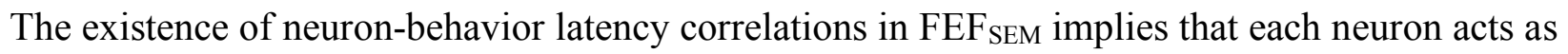
a proxy for many other neurons with correlated response latencies. The predictive power of the latency of a single neuron results from the causal effect of it and its partners. The relationship between LFP and response latency supports this interpretation. To better understand the neural processing that creates these relationships, we next explored the interactions among neuronbehavior latency correlations, neuron-neuron latency correlations, and LFP.

The magnitude of each neuron's neuron-behavior latency correlation is correlated with the coherence of its spikes with the LFP. This observation supports the conclusion that responses in any individual neuron are related to responses in multiple neighbor neurons. For the two example neurons in Figures 5A and B, one (A) has a neural latency that is weakly correlated with the behavioral latency, while the other (B) has neural latency that is strongly correlated with the behavioral latency. The spike-field coherences of the two neurons differed the most in $5-15 \mathrm{~Hz}$ frequency range when we then plotted the z-scored spike field coherences as a function of frequency at $100 \mathrm{~ms}$ after the onset of target motion (Figure 4C).

To quantify this relationship for all $\mathrm{FEF}_{\mathrm{SEM}}$ neurons, we plotted the z-scored spike-field coherence in a time window centered at $106 \mathrm{~ms}$ and a frequency window centered at $7.8 \mathrm{~Hz}$ versus the neuronbehavior latency correlation, revealing a strong relationship (Figure 5D). The Pearson's partial correlation (controlling for firing rate) of 0.43 and 0.36 for monkeys Re and Xt ( $\mathrm{p}=0.0023$ and $0.012, n=50$ and 49 neurons) suggests that the LFP in the frequency range from 5-15 Hz represents

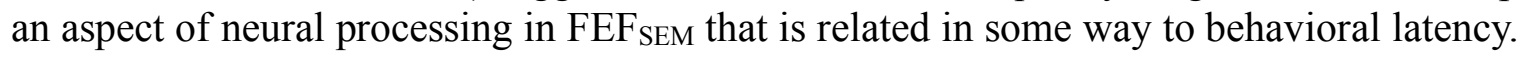

Reanalysis of the neural data collected from area MT for our earlier paper (Lee et al., 2016) reveals that the relationship between the magnitude of spike-field coherence and neuron-behavior latency correlation is weaker in $\mathrm{MT}$ than in $\mathrm{FEF}_{\mathrm{SEM}}$, and the peak in the frequency range from $5-15 \mathrm{~Hz}$ is broader in MT (Figures 5E). The colormap in Figure 5F confirms that the forgoing analysis used the correct frequency band and time interval. Here, each pixel shows the correlation coefficient from graphs like that in Figure 5D for all times and frequencies. The relationship between neuronbehavior latency correlation and LFP is strongest in the frequency range of 5-15 Hz in the time interval from 80-150 ms after the onset of target motion. Comparison with Figure 5G confirms that the relationship between neuron-behavior latency correlation and the LFP is much weaker in MT. These differences support our suggestion that the significance of the 5-15 Hz LFP in FEFSEM is a product of the underlying biology and not simply an artifact of the signal processing, which was identical in the two areas.

The relationships among neuron-behavior latency correlations, behavioral latency, and the low frequency LFP component suggest that the neural synchronization with the LFP could be linked to synchrony in the responses of FEF correlation. We verified this prediction by measuring neuron-neuron latency correlations between pairs of FEFSEM neurons and comparing them with the neuron-behavior latency correlations (Figure 6A, B). We selected 93 pairs of simultaneously recorded neurons that satisfied criteria outlined in the Methods, and calculated trial-by-trial latency correlation following the methods we published before (Lee et al., 2016). Pairwise neuron-neuron latency correlations had a distribution with a mean of 0.04 (Figure 6A). The Pearson correlation is 0.53 between neuron-neuron latency 
correlation and the product of the neuron-behavior latency correlations for the two neurons in the pair (Figure $6 \mathrm{~B}, \mathrm{p}=7.25 \times 10^{-19}, \mathrm{p}=1.2 \times 10^{-4}$ and $1.5 \times 10^{-4}$ for each monkey individually), consistent with expectations if neuron-neuron latency correlations cause neuron-behavior latency correlations. We also found a strong correlation (Pearson's $\rho=0.4, p=2.23 \times 10^{-4}$ ) between neuronneuron latency correlation and the mean of the spike-field coherences for the pair of neurons under study (data not shown).

\section{Other features of neural responses that may contribute to pursuit latency}

It makes sense that the correlated latency of the transient responses of neurons in the FEF a role in determining the latency of pursuit initiation. But, what mechanisms control the neural latencies in FEFSEM? Several possibilities exist: (1) the correlated latency of visual motion inputs from the population response in MT (Lee et al., 2016); (2) the time when a ramp increase in firing rate reaches a threshold ("ramp-to-threshold", Hanes and Schall, 1996); and (3) the amplitude of preparatory activity that ramps up during fixation before the onset of target motion (Darlington et al., 2018). We used analyses of trial-by-trial correlations with behavioral latency to evaluate the variables that might affect neural latencies in FEFSEM.

We observed trial-by-trial correlations between the duration of fixation and behavioral latency and between the amplitude of preparatory activity and behavioral latency, supporting the possibility that the amplitude of preparatory firing rate at the time of target motion onset could contribute to neural latency in $\mathrm{FEF}_{\text {SEM. }}$. The time from the onset of the fixation point to the start of target motion varied between 1100 and $1900 \mathrm{~ms}$ in these experiments, and the trial-by-trial correlation between the duration of fixation and the latency of pursuit was negative $(\mathrm{n}=131$ neurons, average Pearson's correlation across 153 sessions, $r=-0.18$, t-test $\mathrm{p}=2.68 \times 10^{-47}$ ), meaning that longer fixation is associated with shorter behavioral latency. The correlations in the data in the present paper using a 2- or 4-direction pursuit task agreed well with those from the same monkeys in an earlier paper that used a single-direction pursuit task (Figure 7A, C) $\left(\rho=-0.238\right.$, t-test $p=2.25 \times 10^{-130}, \mathrm{n}=95$ sessions with 4 speed/contrast conditions, Darlington et al., 2018).

The correlation between behavioral latency and firing rate at the end of fixation averaged -0.04 , but was quite large for neurons with large modulation of preparatory activity during fixation before the onset of motion. The correlation between the amplitude of preparatory modulation and the preparatory-latency correlation (Figure 7D) was -0.32 (Pearson's correlation, $\mathrm{p}=0.001, \mathrm{n}=99$ neurons), and the neurons from our multiple-direction blocks of trials agreed well with those from single-direction experiments $\left(\rho=-0.32\right.$, t-test $p=4.55 \times 10^{-9}, n=321$ directions collected across 164 neurons, Figure 7B). The wider range of modulation of preparatory activity in the single-direction experiments could reflect either the monkey's greater certainty about the impending target motion, or a difference in the population of sampled neurons.

We conducted two analyses as explicit tests that failed to support the ramp-to-threshold theory of movement latency for FEFSEM.

In the first analysis, we divided all the trials for a single condition of target motion in a single neuron into quintiles according to the latency of pursuit in each trial and computed the timevarying average of firing rate within each quintile. We analyzed the firing rate in the interval from 20-40 ms before the behavioral latency in each quintile (Figure 8A), plotted the firing 
rate as a function of the latency, and fitted the data with a regression line (Figure 8B). The ramp-to-threshold theory posits that the slope of the regression line for these plots should be zero. Across two full populations of FEFSEM neurons, the slopes were close to zero for neurons with relatively weak pursuit-related responses and were strongly positive for neurons with stronger pursuit-related responses (Figure 8C). These two populations of neurons were recorded from the same two monkeys using a single-direction pursuit task with a high-contrast random dot patch target moving at $20 \mathrm{deg} / \mathrm{s}$ (black symbols) or a 2- or 4-direction task (red symbols) with a spot target moving at $10 \mathrm{deg} / \mathrm{s}$.

In the second analysis, we computed the time-varying trajectories of mean eye velocity and firing rate separately for target motions at 2, 10, and $20 \mathrm{deg} / \mathrm{s}$ at high and low contrast for each individual neuron. We then measured the average behavioral latency and the firing rate in the interval from 20-40 ms before the onset of pursuit, plotted the firing rate as a function of the latency. Again, the ramp-to-threshold theory predicts slopes of zero, and we found mostly positive slopes for neurons with large pursuit-related responses and again slopes near zero for neurons with smaller responses. Together, these results do not speak strongly for the ramp-tothreshold theory for the FEF $F_{\text {SEM }}$, especially since the neurons with slopes of zero showed little activity in the interval from $20-40 \mathrm{~ms}$ before the onset of pursuit (Figure $8 \mathrm{~B}$ ).

Contribution of the correlated latency variation in FEF SEM on trial-to-trial variation of smooth pursuit latency

Our data document relationships among a low-frequency component of LFP, neuron-behavior

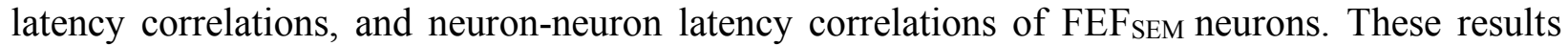

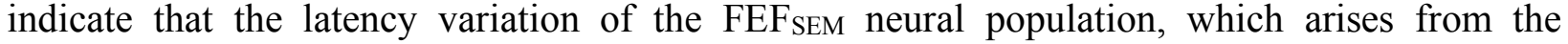
correlated latency variation between neurons, has an impact on behavioral latency variation. Therefore, we asked how much of smooth pursuit latency variation can be accounted for by the correlated neural latency variation in FEF SEM neurons.

We followed the same procedure that we used previously for estimating the contribution of the correlated latency variation of area MT neurons to latency variation in the initiation of smooth pursuit (Lee et al., 2016). The main feature of the procedure is that we need to estimate what we call the "underlying probability of firing" and compute realistic correlations between the latencies of the underlying probabilities of spiking for pairs of model FEFSEM neurons so that we can generate properly correlated model spike trains (details below). We might think of the underlying

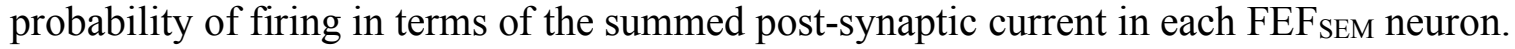

Our analysis has multiple steps.

(1) We calculate the underlying probability of firing from the spiking data for each FEF SEM neuron by averaging spike density across multiple repetitions of the same target motion.

(2) We estimate the standard deviation (SD) of the latency of the underlying firing probability by assuming different values of SD, assembling 100 trials with the underlying firing probability shifted in time according to the SD, laying down simulated spikes with realistic discharge irregularity for each specific neuron, and performing our analysis of the latency of spike trains to estimate the SD of spiking latency. Then, we find the SD of the underlying spiking 
probability that matches the SD of spiking probability we measured from the actual data through the linear regression function between SD of underlying firing probability and estimated SD of simulated spikes. Figure 9A shows the relationship between SD of spiking

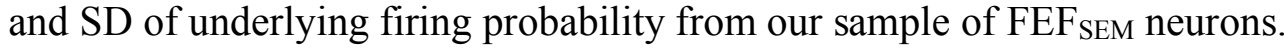

(3) We use a similar procedure to estimate the correlation between the neural latency variation of the underlying firing probability of spiking and the behavioral latency variation. Again, we create model neurons with different degrees of correlation between the latencies of the underlying probability of spiking and of pursuit, lay down simulated spikes with realistic discharge irregularity for each neuron in our sample, and performed our analysis of the trialby-trial correlation between the latencies of the simulated spike trains and the behavior. We then use the neuron-behavior latency correlation for each neuron's actual spikes to estimate its neuron-behavior latency correlation for the underlying probability of firing (Figure 9B). The average neuron-behavior latency correlations were 0.19 for spikes and 0.3 for the underlying probability of firing.

(4) We estimate neuron-neuron latency correlations between underlying probabilities of spiking of pairs of simultaneously recorded neurons using the same procedure outlined above, except with the underlying probability of firing for pairs of neurons. The range of actual correlations is wider for the underlying probability of firing versus the spikes (Figure 9D versus C), and neither shows a compelling relationship to the latency difference between the two neurons. Neuron-neuron latency correlations for spiking and the underlying probability of spiking averaged 0.057 and 0.19 .

(5) Using model parameters based on the distribution of parameters derived for our sample of

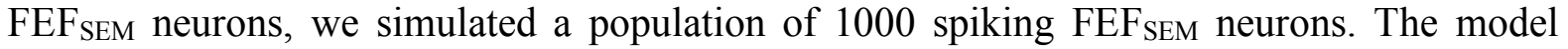
neurons had realistic time dependent shapes of underlying firing probability of spiking, coefficient of variation $(\mathrm{CV})$ of inter-spike interval, neural latency SD, and neuron-neuron latency correlations. We ran the simulation 200 times to represent the responses to 200 trials of target motion. For each trial, we created a population spike density function by convolving each spike train with a Gaussian function having an SD of $10 \mathrm{~ms}$ and then averaging across the responses of 1000 model neurons. We decoded behavioral latency from the population spike density function using the same procedure we had used for estimating latency from the smooth pursuit data.

For the model FEF SEM population, the predicted standard deviation of behavioral latency was 4.6 $\mathrm{ms}$ in the absence of downstream noise and increased as a linear function of Gaussian noise applied downstream (Figure 9E). Therefore, variation in the latency of responses in FEF $F_{\text {SEM }}$ can account alone for at least $40 \%$ of the nominal behavioral latency variation SD of $10 \mathrm{~ms}$ (Osborne et al., 2005). In the experiments reported here, the average SD of pursuit latency was $5.6 \mathrm{~ms}$ (Figure 9E, black dashed line), so that FEFSEM could actually be contributing almost all of the behavioral latency variation.

In the model FEFSEM population, the predicted neuron-behavior latency correlation for underlying firing probability averaged 0.42 in the absence of downstream noise and decreased as a function of the noise added downstream increased (Figure 9E, gray dashed line). In our data, the neuronbehavior latency correlation for the underlying probability of firing in our FEF SEM neurons 
averaged 0.3. When we added random noise from a Gaussian distribution with SD of $3.5 \mathrm{~ms}$ (Figure 9E, vertical line), the SD of predicted behavioral latency was $5.7 \pm 0.3 \mathrm{~ms}$ and the predicted neuron-behavior latency correlation for underlying firing probability was $0.33 \pm 0.02$, both comparable to what we observed in the data. We conclude that under certain assumptions, the latency variation in $\mathrm{FEF}_{\mathrm{SEM}}$ could account for all of the behavioral variation in pursuit latency.

\section{Discussion}

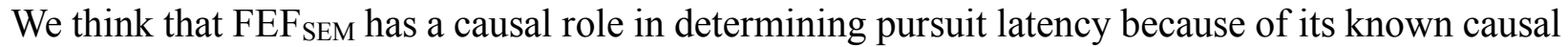
relationship with pursuit eye movements (Brodal, 1980; Gottlieb et al., 1994, 1993; Keating, 1991; Lisberger, 2010; MacAvoy et al., 1991; Ono and Mustari, 2009; Shi et al., 1998; Tanaka and Fukushima, 1998; Tanaka and Lisberger, 2001, 2002b, 2002a). Our analysis reveals four important

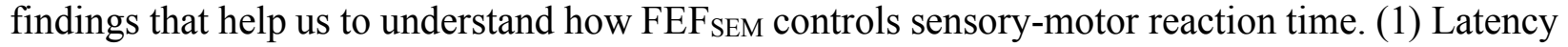
variation of the pursuit-related response in FEFSEM is correlated with latency variation of pursuit behavior and could account for $40-100 \%$ of the variation in behavioral latency. (2) The amplitude of preparatory activity could contribute to the regulation of neural and behavioral latency, but the data do not support the ramp-to-threshold theory strongly for pursuit eye movements. (3) When a neuron's latency variation is highly correlated with the pursuit latency variation, the neuron's latency variation is likely to be correlated with that of neighboring neurons. (4) The latency of

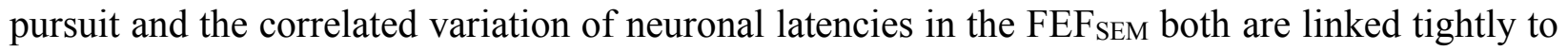
the local field potential in the 5-15 frequency range, and this feature of the LFP seems likely to reflect essential features of the neural control of latency. We propose two components that contribute to response latencies in FEFSEM: the state of preparation and the timing of visual motionrelated inputs. Preparatory and visual motion signals combine to determine the timing of the pursuit-related $\mathrm{FEF}_{\text {SEM }}$ responses that are linked to pursuit reaction times.

\section{Control of pursuit latency}

The control of pursuit latency appears to use a neural mechanism quite different from that for the control of saccade latency. For pursuit, the timing of the overall envelope of firing rate in FEF SEM (present data) and MT (Lee et al., 2016) encodes the latency of the impending pursuit eye movement. The ramp-to-threshold theory that seems to work for saccadic eye movements in other parts of the frontal eye fields (Hanes and Schall, 1996), but not for pursuit eye movements in FEF $_{\text {SEM. }}$. However, the trial-by-trial correlation between the amplitude of preparatory activity and the latency of pursuit raises the possibility that preparatory activity plays a role in determining the latency of the pursuit-related responses in $\mathrm{FEF}_{\mathrm{SEM}}$ and therefore also of pursuit itself.

We have now reported significant trial-by-trial neuron-behavior latency correlations in three major nodes for smooth pursuit eye movement: extrastriate visual area MT, FEFSEM, and the floccular

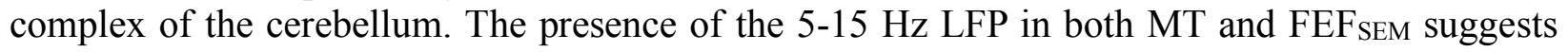
that they aren't completely independent, but simultaneous recordings from MT and FEF SEM will be required to determine the extent to which FEF $_{\text {SEM }}$ and MT control pursuit latency jointly or independently. We admit that our current understanding of the role of FEFSEM in pursuit eye movements somewhat clouds the question of its contribution to pursuit latency. Our correlation analysis indicates that the outputs from MT and FEFSEM both contribute to pursuit initiation, while 
prior data indicate that FEF potentially without actually driving pursuit eye velocity itself. We do not know how a multiplicative modulation would affect pursuit latency. However, one possible answer is that the gain control signal emanating from FEF $_{\mathrm{SEM}}$ is critically important in determining pursuit latency because the visual motion signal from MT cannot initiate the movement when gain is zero.

\section{Origin of correlated variation of FEF}

Our data imply that the correlated latency variation across neurons in FEFsEM contributes to the actual trial-by-trial variation in the latency of pursuit and leads to the impressive neuron-behavior latency correlations we recorded in FEFSEM. Thus, the same framework seems to apply for correlated latencies and response amplitudes in many areas of the brain, including area MT for smooth pursuit eye movements (Hohl et al., 2013; Huang and Lisberger, 2009; Lee et al., 2016; Lee and Lisberger, 2013), area MT for perceptual decisions (Shadlen et al., 1996), FEF SEM for pursuit eye movements (Schoppik et al., 2008), and subcortical vestibular neurons (Liu et al., 2012) and area MSTd (Gu et al., 2014) for heading discrimination. The relationship between the size of the neuron-behavior correlations for a given neuron and its spike-field coherence with the LFP supports strongly the conclusion that neural activity acquires trial-by-trial predictive power for behavioral variation because the neuron under study is part of a population of neurons with correlated variation.

Noise correlations across the relevant population is a necessary, but not sufficient, condition to create neuron-behavior correlations in either latency or response amplitude. In addition, the amount of behavioral variation added downstream from the area under study must be small, because downstream noise will inevitably reduce neuron-behavior correlations (Schoppik et al., 2008). In the neural circuit for pursuit eye movements, it is striking that downstream noise does not eliminate neuron-behavior correlations in either latency or response amplitude. We conclude, as we did before (Osborne et al., 2005), that the trial-by-trial variation in pursuit latency and amplitude is a consequence of correlated noise in cortical areas, and that sub-cortical components of the circuit loyally follow the instructions given by the cortex without judging their veracity. Indeed, the presence of neuron-behavioral correlations limits the amount of noise that can be injected downstream, challenging to some degree the conclusions of Bakhtiari and Pack (2019) about how much noise is created by noise correlations in MT versus downstream from MT.

\section{The possible meaning of $5-15 \mathrm{~Hz}$ LFP}

We found relationships among neuron-neuron latency correlation, neuron-behavior latency correlation, and spike-field coherence in 5-15 Hz frequency range. The three measures covary across neurons that we recorded: a neuron with high synchrony with 5-15 Hz LFP has high neuronneuron latency correlation and high behavior-neuron latency correlation. Their covariation implies that the three factors originate from one mechanism.

The 5-15 Hz frequency range of the LFP overlaps with the alpha range of neural oscillation, but we do not think this is a meaningful piece of information. We find it challenging to reconcile the linkage between the LFP wave and pursuit initiation with the relationship of alpha wave to feedback signaling (Michalareas et al., 2016), attention (Saalmann et al., 2012), attentional gating (Foxe and Snyder, 2011) or working memory (Gevins et al., 1997). We also note but cannot 
reconcile our findings with the LFP suppression in the beta range that precedes arm movement (Best et al., 2017), or the correlation between beta power and reaching reaction time in the primary motor cortex (Khanna and Carmena, 2017). A previous study reported a traveling beta wave in macaque area V4 that is correlated with the parameters of saccadic eye movements (Zanos et al., 2015), including an interesting phase relationship between beta frequency LFP and the timing of saccade offset. Our findings differ from this study in the specific frequency of LFP (beta vs. 5-15 $\mathrm{Hz}$ ), the behavior (saccade vs. smooth pursuit), the brain area (area V4 vs. FEFSEM), and the time epochs for the correlation (after saccade offset vs. before pursuit initiation). In many ways, the

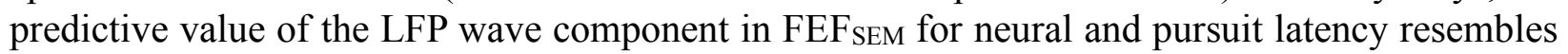
that for gamma synchrony in the LFP in area MT for neuron-neuron spike count correlations (Lee and Lisberger, 2013). It is curious that latency and spike rate are related to LFP in such different frequency ranges.

Finally, we are struck by the fact that the LFP wave in the $5-15 \mathrm{~Hz}$ frequency range shifts in time in parallel with pursuit latency, and that its phase is so well correlated with pursuit latency on a trial-to-trial basis. We view the $5-15 \mathrm{~Hz}$ frequency components in the LFP as a signature of critical neural activity that gets synchronized in the FEFSEM to facilitate initiation of a pursuit eye movement. It could reflect internal activity in $\mathrm{FEF}_{\mathrm{SEM}}$, could be a correlate of a trigger for pursuit initiation from another node in the pursuit circuit, or might be simply a consequence of latency correlations in inputs from area MT that report the sensory estimate of target speed and direction. We suspect that understanding the latency-related component of the LFP will prove to be a key in unraveling that neural mechanisms that determine behavioral latency in our particular sensorymotor system, and possibly in other systems as well.

\section{Materials and Methods}

Two adult male rhesus monkeys were trained to pursue a circular dot that executed a step-ramp trajectory of target position (Rashbass, 1961), in exchange for juice or water reward. Before training, we performed two separate surgeries using sterile procedure. In the first surgery, a head holder was implanted on the skull for head restraint, and a stainless-steel chamber was implanted on the skull, over the cross-section of arcuate sulcus and central sulcus. In the second surgery, a scleral search coil was implanted in one eye (Ramachandran and Lisberger, 2005). After finishing behavioral training, we performed a craniotomy inside the chamber for introducing quartzinsulated platinum/iridium electrodes into the brain. All experiments were conducted at Duke University using methods that had been approved in advance by the Institutional Animal Care and Use Committee at Duke University. Methods conformed to the National Institutes of Health Guide for the Care and Use of Laboratory Animals. The latency distribution for neurons and the relationship between spikes and LFP in area MT were obtained by reanalyzing the data reported in previous papers (Lee et al., 2016; Lee and Lisberger, 2013). We also performed a few analyses on data collected from the same 2 monkeys in previous experiments on FEFSEM (Darlington et al., 2018). 


\section{$\underline{\text { Data acquisition }}$}

Visual stimuli were presented on a gamma-corrected 24-inch CRT color monitor (Sony Trinitron), whose spatial resolution was 2304 by 1440 pixels and vertical refresh rate was $80 \mathrm{~Hz}$. The monitor was placed $60 \mathrm{~cm}$ from the monkey and the screen covered 44 by 29 deg of horizontal and vertical visual fields. Accurate measurement of visual motion onset timing is guaranteed by the post-hoc data collection using photodiode. Since we did not use the photodiode system during recording experiments, all the visual motion onset timing has been corrected by the reference data collected after the experiments. Horizontal and vertical eye position and velocity were sampled at $1 \mathrm{kHz}$. Eye velocity was obtained by differentiating and low-pass filtering eye position at a cut-off frequency of $25 \mathrm{~Hz}$ using an analog circuit.

During experiments, we slowly lowered one to three electrodes or tetrodes in the FEF SEM $_{\text {and }}$ recorded spikes and local field potentials (LFP) using the Tetrode Mini-matrix System (Thomas Recording $\mathrm{GmbH}$ ). The input impedance and gain of the Mini-matrix preamplifier were $1 \mathrm{G} \Omega$ and x19 individually. The input impedance of the preamplifier was sufficiently higher than the impedance of the single electrodes and tetrodes that we used (Thomas Recording GmbH, 2-4 M $\Omega$ and 1-2 M $\Omega$ individually), ensuring that our recordings would be relatively free from phase distortion in low frequency LFPs (Nelson et al., 2008). For LFPs, signals were low-pass filtered with a cut-off frequency of $170 \mathrm{~Hz}$ and digitized at a sampling rate of $2 \mathrm{kHz}$. For action potentials, signals were high-pass filtered with a cut-off frequency of $150 \mathrm{~Hz}$ and those that crossed a preset threshold were digitized at a sampling rate of $40 \mathrm{kHz}$. Digitization, filtering, and amplification of signals were done with a Plexon MAP system (Plexon Inc.).

We isolated single neural spikes online, using a window discriminator for analyzing and displaying neural response properties. We performed more accurate off-line discrimination of single neural responses later, using spike waveforms that exceeded a certain threshold. We took exceptional care in spike sorting because any significant sorting errors might work as an added noise in trialby-trial correlation analysis. First, we performed initial spike sorting using principal component analysis. Then, we refined the sorting by visually inspecting the waveforms in a smaller time chunk, to detect any waveform changes caused by the slow drift of electrodes. We used Plexon Offline Sorter (Plexon Inc.) for all the offline spike sorting procedure. Sorted spikes were converted to time stamps with a time resolution of $1 \mathrm{~ms}$ and were inspected again visually to look for obvious sorting errors.

\section{Experimental design}

We recorded neural activity in $\mathrm{FEF}_{\text {SEM }}$ while two rhesus monkeys were engaged in smooth pursuit

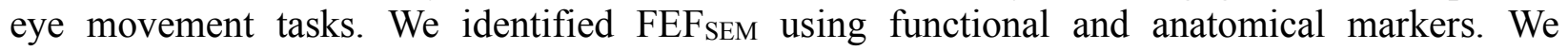
implanted a recording chamber over the arcuate sulcus so that we could have perpendicular access to the rostral bank of the sulcus. As we advanced electrodes, we first looked for the area that shows oculomotor responses. We then advanced the electrodes past saccade-related neurons until we found neurons that were strongly modulated by smooth pursuit eye movements. Then we isolated single neuron responses and performed two experiments.

In the first experiment, we tested the direction tuning of the neurons under study during smooth pursuit eye movement. We randomly interleaved saccade trials and pursuit trials, where saccade 
trials required the monkeys to make 8 directions ( 45 deg interval) of 15 deg amplitude saccade eye movements and pursuit trials required them to make 8 directions (45 deg interval) of pursuit eye movements at $15 \mathrm{deg} / \mathrm{s}$. We used a white circular dot as a saccade target and random dot stimulus and/or grating as pursuit targets. The random dot stimulus was made from white and black dots so as for us to control luminance contrast of the stimulus (see Yang et al., 2012). Typically, luminance contrast of the random dot stimulus was $100 \%$ and contrast of the grating stimulus was $12 \%$. We usually used both stimuli, but either sufficed for determining the direction tuning of FEF SEM neurons. All the visual stimuli were presented on a gamma corrected, gray background. We obtained saccade and pursuit direction tuning curves online using a least-square fitting method and used the tuning curves to choose the preferred pursuit direction of the neurons under recording, and to test whether the neurons were more-strongly tuned to smooth pursuit or saccadic eye movement. Only the 133 neurons with larger responses during pursuit than during saccades were admitted for further analysis.

In the second experiment, we recorded neural responses while monkeys tracked a yellow circular dot that moved in a step-ramp trajectory. Typically, we choose two pursuit directions that provided good compromises among the preferred directions of the neurons under study and one target speed $(10 \mathrm{deg} / \mathrm{s}$ or $20 \mathrm{deg} / \mathrm{s})$; sometimes we used both target speeds and more than two directions. The size of the step in the step-ramp target motion was 1 and 3 deg for target speeds of 10 and $20 \mathrm{deg} / \mathrm{s}$. After the experiment, we screened all trials visually and excluded from further analysis any pursuit trials that contained saccades or microsaccades in the interval from $100 \mathrm{~ms}$ before to $210 \mathrm{~ms}$ after target motion onset.

\section{Estimation of pursuit latency and FEF $\underline{\text { SEM-pursuit latency correlation }}$}

We used an objective method that was developed in our previous study to quantify the latency of pursuit for each trial(Lee et al., 2016; Lee and Lisberger, 2013). Briefly, we computed the average horizontal and vertical eye velocities across trials and used the averages from $20 \mathrm{~ms}$ before to 80 $\mathrm{ms}$ after the initiation of pursuit as templates. Here, we used the initial $80 \mathrm{~ms}$ of smooth pursuit, instead of $100 \mathrm{~ms}$ to include more trials in the analysis. The overall result for pursuit latency was the same whether we used $80 \mathrm{~ms}$ or $100 \mathrm{~ms}$ for the analysis. We shifted and scaled the templates in every trial and estimated the best match using the least-square method. The best time shift defined the pursuit latency in each trial. We included a trial in the further analysis only if the bestfitted estimate accounted for more than $80 \%$ of eye-velocity variance. We proceeded to further analysis only if 80 or more trials passed this test.

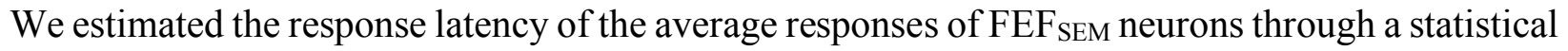
method. First, we obtained spike density functions by convolving spike trains with a Gaussian window of a $10 \mathrm{~ms}$ standard deviation and averaging the individual spike density functions across trials. Then, we set a baseline by averaging neural activity in the interval $\pm 25 \mathrm{~ms}$ from motion onset. If the average PSTH between $30 \mathrm{~ms}$ and $300 \mathrm{~ms}$ from motion onset exceeded the baseline activity plus $20 \%$ of the standard deviation of the baseline and continued to do so for more than $50 \mathrm{~ms}$, the first time bin of the $50 \mathrm{~ms}$ was selected as the latency of the average firing rate.

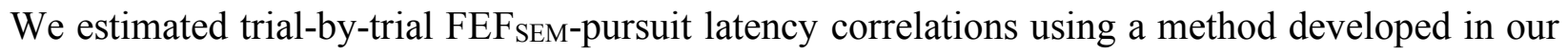
previous study (Lee et al., 2016). First, we excluded any neurons whose responses in the interval from 1 to $100 \mathrm{~ms}$ after neural response latency failed to reach 20 spikes/s, leaving $99 \mathrm{FEF}$ SEM 
neurons from the two monkeys for further analysis. Then, we selected one pursuit condition per neuron that provided the largest average response across the interval from 1 to $100 \mathrm{~ms}$ after the time chosen as the neural response latency. By taking advantage of the fact that the correlation coefficient is a regression slope of Z-transformed data (Rodgers and Nicewander, 1988), we obtained the correlation by taking a geometrical mean of the two regression coefficients - one coefficient with pursuit latency as independent variable and neural latency as dependent variable, and the other with neural latency as independent variable and pursuit latency as dependent variable.

To calculate a regression coefficient when pursuit latency is the independent variable, we divided the trials from each neuron into five equal-sized groups (quintiles) according to the estimated pursuit latency. We computed the spike density functions by averaging across the trials in each quintile of trials. Using the mean spike density function for all trials as a template, we estimated the best fitting values of time shift for each quintile by shifting and scaling the template to match the quintile's spike density. Then, we calculated the regression coefficient for the estimated time shift of the mean spike density as a function of the mean pursuit latency for each quintile.

To calculate a regression coefficient when neuronal latency is an independent variable, we used an iterative process to estimate neuronal latency in individual trials. Using the mean spike density function for all trials as the template, we estimated time shifts and scaling factors for the spike density from each individual trial. We then updated the template using a Bayesian procedure (Bollimunta et al., 2007) and repeated the estimation procedure two more times. Then, we divided trials into quintiles according to the final estimates of neural latency and computed mean spike density function for each quintile of trials. We estimated the five best fitting values of time shifts by shifting and scaling the template along the mean spike density function of each quintile. Finally, we calculated the regression coefficient for pursuit latency as a function of the estimated neural latencies for the 5 quintiles. We obtained the Pearson's correlation coefficient by taking geometrical mean of the two regression coefficients. We applied the same procedure using the responses of pairs of neurons to estimate neuron-neuron latency correlations. We started from 134 unique pairs, and again selected pairs with the average of the two average responses across the interval from 1 to $100 \mathrm{~ms}$ after each neural latency exceeds 20 spikes/s, which leaves 93 pairs in the further analysis.

\section{Local field potential analysis}

Spike-field coherence. We preprocessed the LFP data with a Butterworth filter to remove $60 \mathrm{~Hz}$ line noise, using a function in the Fieldtrip MATLAB toolbox (Oostenveld et al., 2011). Then we analyzed the 'spike-field coherence' between the spikes and the LFP recorded from the same electrode. We used the Chronux MATLAB toolbox (Bokil et al., 2010) for all the spike-field coherence analysis. For the low frequencies $(1-20 \mathrm{~Hz})$, we used a Hanning window as a taper. For frequency ranges between 20 and $170 \mathrm{~Hz}$, we used five spheroidal tapers with $15 \mathrm{~Hz}$ of spectral smoothing. We calculated the spike-field coherence in $150 \mathrm{~ms}$ sliding windows with a step size of $10 \mathrm{~ms}$. We calculated coherence and transformed the coherence using the variance stabilization method (Bokil et al., 2007). To correct a potential confound that can occur at the low frequencies due to the transient responses (Jarvis and Mitra, 2001), we shuffled trials and calculated the variance stabilized coherences 1000 times and generated the "null distributions" of coherences across frequencies. Then we obtained a z-score as a function of frequency using the shuffle analysis as in our previous study (Lee and Lisberger, 2013). 
LFP phase-pursuit latency correlation. First, we used a $6^{\text {th }}$ order Butterworth filter to bandpass filtered the LFP and isolated frequency in bandwidths of $2 \mathrm{~Hz}$ and $1 \mathrm{~Hz}$ steps from 0 to $160 \mathrm{~Hz}$. We obtained power and phase information from each bandpass filtered component by applying Hilbert transformation. Then, across trials and for each millisecond within trial, we calculated the correlation between instantaneous phase/power at that instant and estimated pursuit latency (Figures 2A, B). From the correlation map, we selected the frequency range that maximized the correlation, isolated the LFP in the $5-15 \mathrm{~Hz}$ range using a $6^{\text {th }}$ order Butterworth filter, and calculated the correlation between instantaneous phase/power and pursuit latency after Hilbert transformation. Because phase information has a circular form and pursuit latency has a linear form, we used the correlation coefficient calculation for circular and linear data (Berens, 2009).

\section{Underlying probability of spiking and computer simulations}

As we showed in our previous study (Lee et al., 2016), variation in neural latency has two components, one created by variation in the timing of the underlying probability of spiking, and the other created by the stochastic nature of spike timing. To estimate the SD of the latency of the underlying probability of spiking, we used a computational simulation approach separately for each FEF SEM neuron. (1) We calculated the coefficient of variation (CV) using inter-spike intervals in a time window from 100 to $300 \mathrm{~ms}$ after the average neural latency. (2) We recalculated the peristimulus time histogram to estimate a template for the underlying probability of spiking by aligning each trial according to its estimated neural latency and applying a Gaussian filter (3 ms SD) to the re-aligned PSTH. (3) In different runs where the SD of the latency variation was an independent variable, we jittered the template of underlying probability in latency across trials using random values from a Gaussian distribution with a mean of zero and SD chosen from predetermined values $(1,2,4,6,8,10,12,16,20,24,28,32,36,40)$. (4) For each run, we used the neuron's $\mathrm{CV}$ as the basis for creating simulated spike trains that follow a renewal Gamma process (Brown et al., 2002; Koyama and Shinomoto, 2005). (5) We estimated latency SD from the simulated spike train using the same procedure that we used for real data. (6) We used the simulated relationship between latency SD for spikes and underlying probability of firing to estimate the actual latency SD of the underlying probability given the latency SD measured from each neuron's spiking. In this step, we included neurons only if the regression explained more than $40 \%$ of the variance, reducing the number of neurons included in the further analysis from 99 to 94 (Figure 7A).

Once we estimate SD of latency for spiking and underlying firing probability, we can estimate latency correlation between underlying probability and behavior or between two underlying probabilities.

$$
\begin{gathered}
R_{\text {prob,behavior }} \approx R_{\text {spikes,behavior } \frac{\sigma\left(L_{\text {spikes }}\right)}{\sigma\left(L_{\text {prob }}\right)}} \\
R_{\text {prob1,prob } 2} \approx R_{\text {spikes } 1, \text { spikes } 2} \frac{\sigma\left(L_{\text {spikes } 1}\right) \sigma\left(L_{\text {spikes } 2}\right)}{\sigma\left(L_{\text {prob } 1}\right) \sigma\left(L_{\text {prob } 2}\right)}
\end{gathered}
$$

Where $L$ represents latency. Following the two equations, we estimated latency correlation between underlying probability and behavior, and latency correlation between the two underlying probabilities. When we estimated latency correlation between underlying probability and behavior, 
we only selected neurons whose estimated latency SDs of underlying probability of spiking are the same or bigger than one, to avoid over-estimation of the correlation. This resulted in sample reduction for the estimation of latency correlation between underlying probability and behavior, from 94 to 76 .

Computer simulations. To estimate how much of pursuit latency variation could originate from FEF $_{\text {SEM, }}$, we conducted a computational analysis. We simulated a population of 1000 spiking

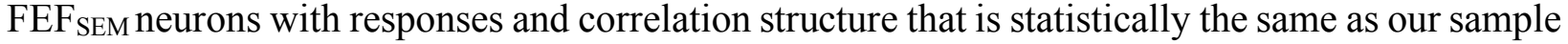
of FEF SEM neurons. We used re-aligned time-varying mean spike density functions of our FEF SEM recordings to determine the underlying probability of spiking. We also randomly selected the latency SD of the underlying firing probability of spiking from a Gamma distribution fitted to our observed distribution of latency SD with a shape parameter к of 1.39 and scale parameter $\theta$ of 10.16. For each model neuron, we selected a value of SD from the Gamma distribution, drew 200 latencies from a Gaussian distribution with that SD, and shifting the underlying firing probability of spiking in time by those values for our 200 simulated trials. In the population of 1000 model FEF $_{\text {SEM }}$ neurons, we also retained the structure of the neuron-neuron latency correlations for the underlying probability of firing derived from our data: the distribution of pairwise latency correlation followed a Gaussian distribution with a mean of 0.19. For each trial in each model neuron, we modeled the inter-spike interval distribution by using the shape of the latency-jittered time dependent underlying firing probability to scale a renewal Gamma process based on the CV of inter-spike interval distribution in our data (Brown et al., 2002; Koyama and Shinomoto, 2005). We estimated the spike density of each simulated neuron by applying a Gaussian filter with SD of $10 \mathrm{~ms}$ and computed the population average across the 1000 simulated neurons' spike density functions in each trial. From these population spike density functions, we estimated the latency in individual trials. We simulated the added downstream noise by generating random number that follows a Gaussian distribution, with zero mean and SD ranged from 0 to $10 \mathrm{~ms}$ in $0.5 \mathrm{~ms}$ step. We repeated the whole simulation process 10 times to calculate the mean and SD of simulated neuron-behavior latency correlation for the underlying firing probability and SD of simulated latencies. 


\section{References}

Bakhtiari S, Pack CC 2019 Functional specialization in the middle temporal area for smooth pursuit initiation. MNI Open Res. 2: 1-26. https://doi.org/10.12688/mniopenres.12806.2

Berens P. 2009. CircStat: A MATLAB toolbox for circular statistics. J Stat Softw 31:1-21. doi:10.1002/wics. 10

Best MD, Suminski AJ, Takahashi K, Brown KA, Hatsopoulos NG. 2017. Spatio-temporal patterning in primary motor cortex at movement onset. Cereb Cortex 27:1491-1500. doi:10.1093/cercor/bhv327

Bokil H, Andrews P, Kulkarni JE, Mehta S, Mitra PP. 2010. Chronux: a platform for analyzing neural signals. J Neurosci Methods 192:146-51. doi:10.1016/j.jneumeth.2010.06.020

Bokil H, Purpura K, Schoffelen J-M, Thomson D, Mitra P. 2007. Comparing spectra and coherences for groups of unequal size. J Neurosci Methods 159:337-45. doi:10.1016/j.jneumeth.2006.07.011

Bollimunta A, Knuth KH, Ding M. 2007. Trial-by-trial estimation of amplitude and latency variability in neuronal spike trains. $J$ Neurosci Methods 160:163-70. doi:10.1016/j.jneumeth.2006.08.007

Brodal P. 1980. The cortical projection to the nucleus reticularis tegmenti pontis in the rhesus monkey. Exp Brain Res 38:19-27.

Brown EN, Barbieri R, Ventura V, Kass RE, Frank LM. 2002. The time-rescaling theorem and its application to neural spike train data analysis. Neural Comput 14:325-346. doi: $10.1162 / 08997660252741149$

Darlington TR, Beck JM, Lisberger SG. 2018. Neural implementation of Bayesian inference in a sensorimotor behavior. Nat Neurosci 21:1442-1451. doi:10.1038/s41593-018-0233-y

Foxe JJ, Snyder AC. 2011. The role of alpha-band brain oscillations as a sensory suppression mechanism during selective attention. Front Psychol 2:1-13. doi:10.3389/fpsyg.2011.00154

Gevins A, Smith ME, McEvoy L, Yu D. 1997. High-resolution EEG mapping of cortical activation related to working memory: Effects of task difficulty, type of processing, and practice. Cereb Cortex 7:374-385. doi:10.1093/cercor/7.4.374

Gold JI, Shadlen MN. 2007. The neural basis of decision making. Annu Rev Neurosci 30:53574. doi:10.1146/annurev.neuro.29.051605.113038

Gottlieb JP, Bruce CJ, MacAvoy MG. 1993. Smooth eye movements elicited by microstimulation in the primate frontal eye field. J Neurophysiol 69:786-799. 
Gottlieb JP, MacAvoy MG, Bruce CJ. 1994. Neural responses related to smooth-pursuit eye movements and their correspondence with electrically elicited smooth eye movements in the primate frontal eye field. $J$ Neurophysiol 72:1634-53.

Gu Y, Angelaki DE, DeAngelis GC. 2014. Contribution of correlated noise and selective decoding to choice probability measurements in extrastriate visual cortex. Elife e02670. doi:10.7554/eLife. 02670

Hanes DP, Schall JD. 1996. Neural control of voluntary movement initiation. Science 274:42730.

Hohl SS, Chaisanguanthum KS, Lisberger SG. 2013. Sensory population decoding for visually guided movements. Neuron 79:167-179. doi:10.1016/j.neuron.2013.05.026

Huang X, Lisberger SG. 2009. Noise correlations in cortical area MT and their potential impact on trial-by-trial variation in the direction and speed of smooth-pursuit eye movements. $J$ Neurophysiol 101:3012-30. doi:10.1152/jn.00010.2009

Jarvis MR, Mitra PP. 2001. Sampling properties of the spectrum and coherency of sequences of action potentials. Neural Comput 13:717-49.

Keating EG. 1991. Frontal eye field lesions impair predictive and visually-guided pursuit eye movements. Exp Brain Res 86:311-323. doi:10.1007/BF00228954

Khanna P, Carmena JM. 2017. Beta band oscillations in motor cortex reflect neural population signals that delay movement onset. Elife 6:1-31. doi:10.7554/eLife.24573

Koyama S, Shinomoto S. 2005. Empirical Bayes interpretations of random point events. $J$ Phys A Math Gen 38:L531-L537. doi:10.1088/0305-4470/38/29/L04

Lee J, Joshua M, Medina JF, Lisberger SG. 2016. Signal, noise, and variation in neural and sensory-motor latency. Neuron 90:165-176. doi:10.1016/j.neuron.2016.02.012

Lee J, Lisberger SG. 2013. Gamma synchrony predicts neuron-neuron correlations and correlations with motor behavior in extrastriate visual area MT. J Neurosci 33:19677-88. doi:10.1523/JNEUROSCI.3478-13.2013

Lisberger SG. 2010. Visual guidance of smooth-pursuit eye movements: sensation, action, and what happens in between. Neuron 66:477-491. doi:10.1016/j.neuron.2010.03.027

Lisberger SG, Movshon JA. 1999. Visual motion analysis for pursuit eye movements in area MT of macaque monkeys. J Neurosci 19:2224-46.

Liu S, Gu Y, Deangelis GC, Angelaki DE. 2012. Choice-related activity and correlated noise in subcortical vestibular neurons. Nat Neurosci 1-11. doi:10.1038/nn.3267

MacAvoy MG, Gottlieb JP, Bruce CJ. 1991. Smooth-pursuit eye movement representation in the primate frontal eye field. Cereb Cortex 1:95-102. doi:10.1093/cercor/1.1.95 
Michalareas G, Vezoli J, van Pelt S, Schoffelen JM, Kennedy H, Fries P. 2016. Alpha-beta and gamma rhythms subserve feedback and feedforward influences among human visual cortical areas. Neuron 89:384-397. doi:10.1016/j.neuron.2015.12.018

Nelson MJ, Pouget P, Nilsen E a, Patten CD, Schall JD. 2008. Review of signal distortion through metal microelectrode recording circuits and filters. J Neurosci Methods 169:14157. doi:10.1016/j.jneumeth.2007.12.010

Newsome WT, Wurtz RH, Komatsu H. 1988. Relation of cortical areas MT and MST to pursuit eye movements. II. Differentiation of retinal from extraretinal inputs. $J$ Neurophysiol 60:604-20.

Ono S, Mustari MJ. 2009. Smooth pursuit-related information processing in frontal eye field neurons that project to the NRTP. Cereb Cortex 19:1186-1197. doi:10.1093/cercor/bhn166

Oostenveld R, Fries P, Maris E, Schoffelen JM. 2011. FieldTrip: Open source software for advanced analysis of MEG, EEG, and invasive electrophysiological data. Comput Intell Neurosci 2011. doi:10.1155/2011/156869

Osborne LC, Lisberger SG, Bialek W. 2005. A sensory source for motor variation. Nature 437:412-416. doi:10.1038/nature03961

Ramachandran R, Lisberger SG. 2005. Normal performance and expression of learning in the vestibulo-ocular reflex (VOR) at high frequencies. $J$ Neurophysiol 93:2028-38. doi:10.1152/jn.00832.2004

Rashbass C. 1961. The relationship between saccadic and smooth tracking eye movements. $J$ Physiol 159:326-338. doi:10.1016/0196-9781(92)90172-Y

Rodgers JL, Nicewander WA. 1988. Thirteen ways to look at the correlation coefficient. Am Stat 42:59. doi:10.2307/2685263

Saalmann YB, Pinsk M a., Wang L, Li X, Kastner S. 2012. The pulvinar regulates information transmission between cortical areas based on attention demands. Science (80- ) 337:753756. doi:10.1126/science. 1223082

Schoppik D, Nagel KI, Lisberger SG. 2008. Cortical mechanisms of smooth eye movements revealed by dynamic covariations of neural and behavioral responses. Neuron 58:248-260. doi:10.1016/j.neuron.2008.02.015

Shadlen MN, Britten KH, Newsome WT, Movshon JA. 1996. A computational analysis of the relationship between neuronal and behavioral responses to visual motion. $J$ Neurosci 16:1486-1510.

Shi D, Friedman HR, Bruce CJ. 1998. Deficits in smooth-pursuit eye movements after muscimol inactivation within the primate's frontal eye field. $J$ Neurophysiol 80:458-464. 
Tanaka M, Fukushima K. 1998. Neuronal responses related to smooth pursuit eye movements in the periarcuate cortical area of monkeys. J Neurophysiol 80:28-47.

Tanaka M, Lisberger SG. 2002a. Enhancement of multiple components of pursuit eye movement by microstimulation in the arcuate frontal pursuit area in monkeys. $J$ Neurophysiol 87:802818.

Tanaka M, Lisberger SG. 2001. Regulation of the gain of visually guided smooth-pursuit eye movements by frontal cortex. Nature 409:191-4. doi:10.1038/35051582

Tanaka M, Lisberger SGS. 2002b. Role of arcuate frontal cortex of monkeys in smooth pursuit eye movements . I . Basic response properties to retinal image motion and position. $J$ Neurophysiol 87:2684-2699.

Yang J, Lee J, Lisberger SG. 2012. The interaction of Bayesian priors and sensory data and its neural circuit implementation in visually guided movement. $J$ Neurosci 32:17632-45. doi:10.1523/JNEUROSCI.1163-12.2012

Zanos TP, Mineault PJ, Nasiotis KT, Guitton D, Pack CC. 2015. A sensorimotor role for traveling waves in primate visual cortex. Neuron 85:615-627. doi:10.1016/j.neuron.2014.12.043 


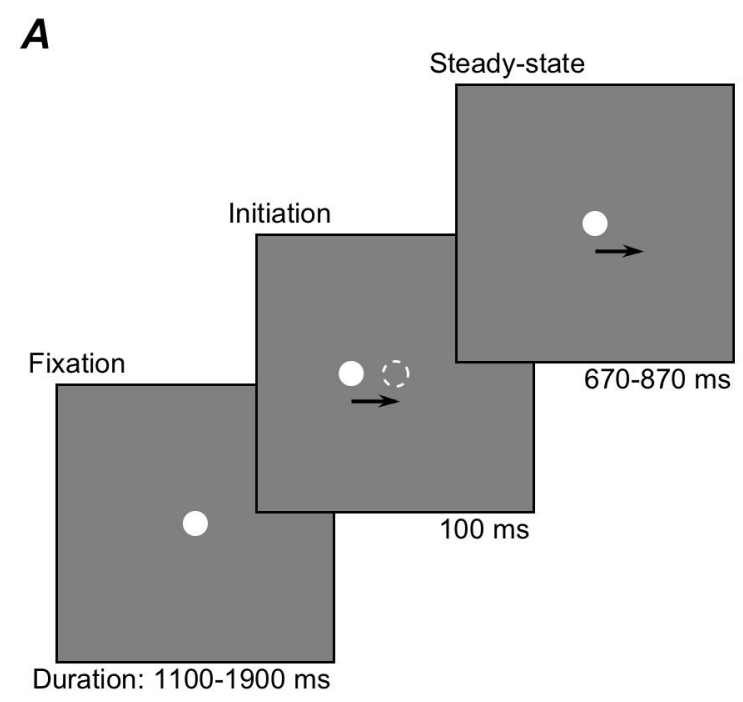

B

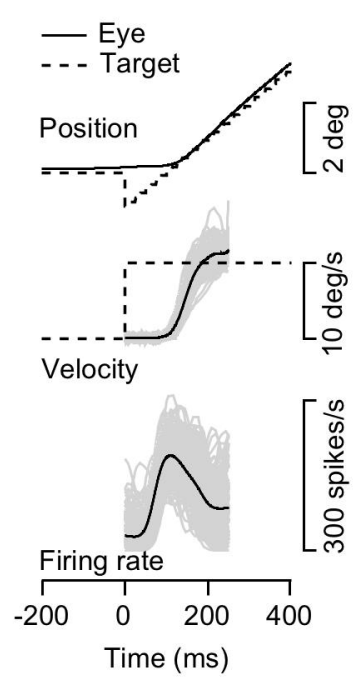

Figure 1. Structure of the task and example traces of step-ramp target motion and smooth pursuit eye movements. A: The three components of step-ramp target motion. B: Superimposed eye and target motion and spike density function traces. Dashed and continuous traces in the top two panels show target and eye motion. The black and gray traces for eye velocity and firing rate show averages across trials and data from individual trials. 

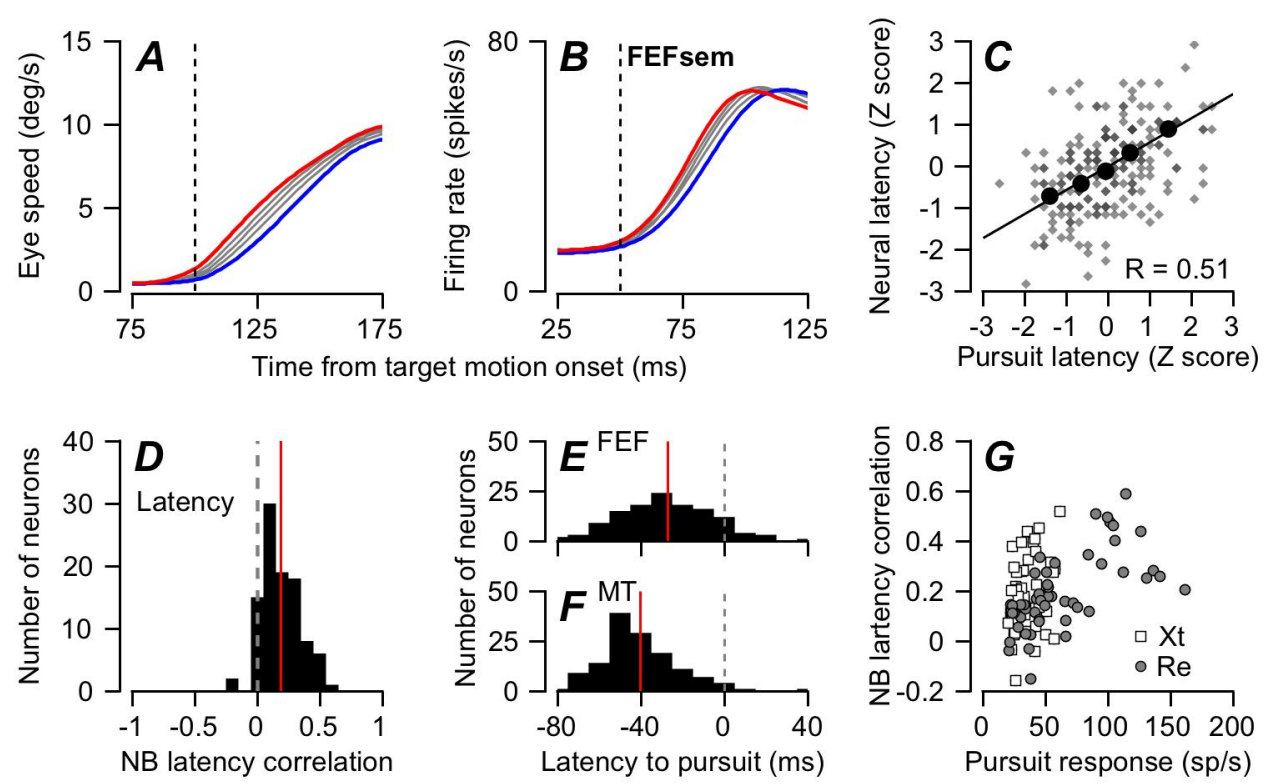

Figure 2. Trial-by-trial relationships between latency variation in pursuit behavior and firing of FEF SEM neurons. A: Eye speed versus time during the initiation of pursuit. B: Firing rate of an example FEF SEM neuron in the same intervals. In A-B, the five traces in each panel show data for quintiles of trials sorted by the latency of pursuit. Red and blue traces show data for the shortest and longest latency group. Gray colors show data for the middle groups. C: Plot of z-scored neural latency versus z-scored pursuit latency for an example neuron. Black circles show data for the 5 quintiles based on pursuit latency and small gray symbols show measurements for individual trials. Line was obtained by regression. D: Distribution of response latency-pursuit latency correlations for $99 \mathrm{FEF}_{\mathrm{SEM}}$ neurons. E, F: Distributions of latency from neural responses in MT and FEF SEM $_{\text {to }}$

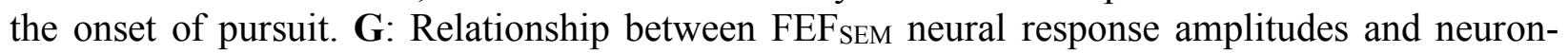
behavior latency correlations. The correlation between neural responses and neuron-behavior latency correlations was 0.46 (Pearson's correlation, $p=1.4 \times 10^{-6}$ ). Different symbols in $\mathbf{G}$ show data recorded in different monkeys. 

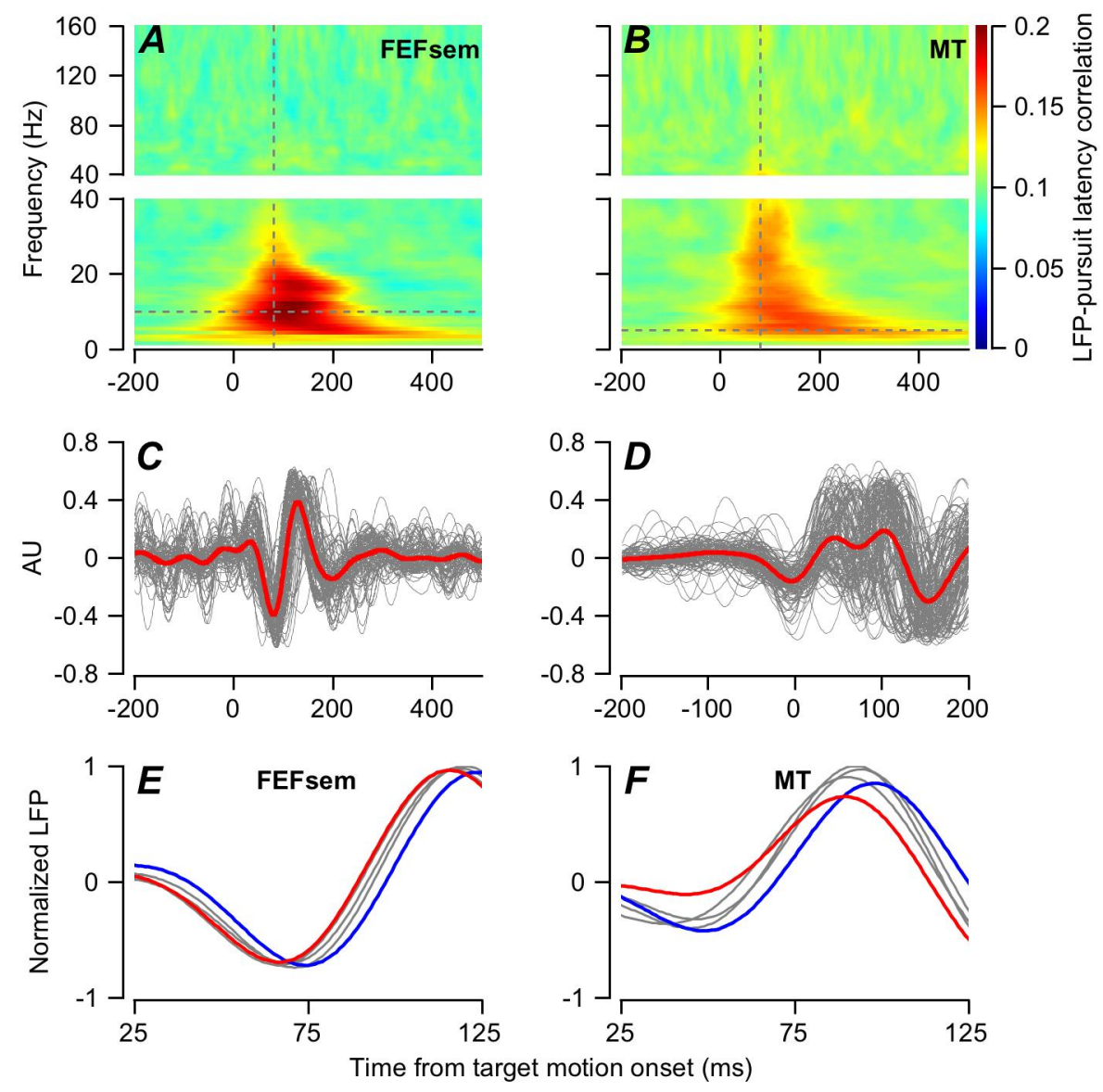

Figure 3. Local field potential and its correlation with the latency of pursuit. A, B: The color of each pixel plots the trial-by-trial correlations between pursuit latency and the phase of the LFP averaged across 123 sites in $\mathrm{FEF}_{\text {SEM }}(\mathbf{A})$ and 138 sites in MT (B) as function of LFP frequency in $1 \mathrm{~Hz}$ bands and time from the onset of target motion. C, D: Traces for local field potential filtered with a bandpass of $5-15 \mathrm{~Hz}$ for $\mathrm{FEF}_{\mathrm{SEM}}(\mathbf{C})$ and MT (D). Red and gray traces show averages across all the recordings and data from individual average recordings for the FEFSEM. E, F: LFP sorted into quintiles according to pursuit latency, after normalizing individual filtered LFPs from individual trials by the maximum peak to trough distance. Red and blue traces show data for the shortest and longest latency group. Gray colors show data for the middle groups. Example neurons recorded in $\mathrm{FEF}_{\mathrm{SEM}}(\mathbf{E})$ and $\mathrm{MT}(\mathbf{F})$. 


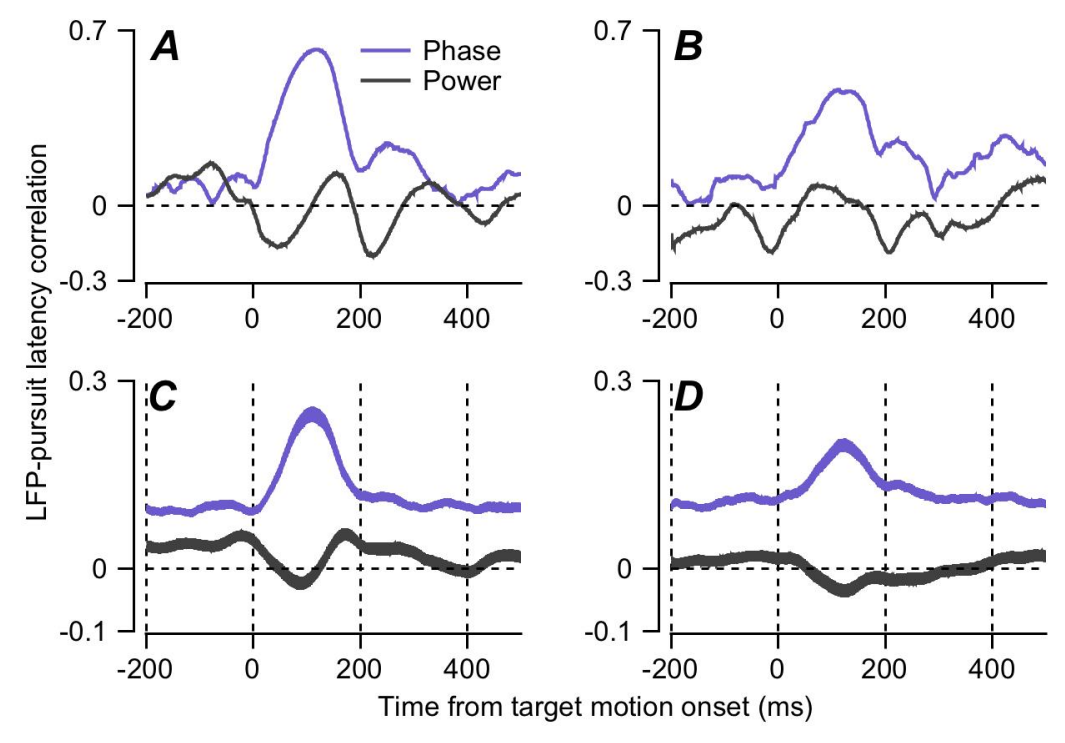

Figure 4. Quantitative analysis of the relationship between local field potential and pursuit latency. Graphs show example recordings in FEF $\mathrm{SEM}$ (A) and MT (B) and averaged across all recording sites in $\mathrm{FEF}_{\mathrm{SEM}}(\mathbf{C})$ and $\mathrm{MT}(\mathbf{D})$ as a function of the time during the wave. Blue and black traces show correlations of the LFP phase versus power, and the ribbons around the traces in $\mathbf{C}$ and $\mathbf{D}$ show the standard error of the mean across recordings. 

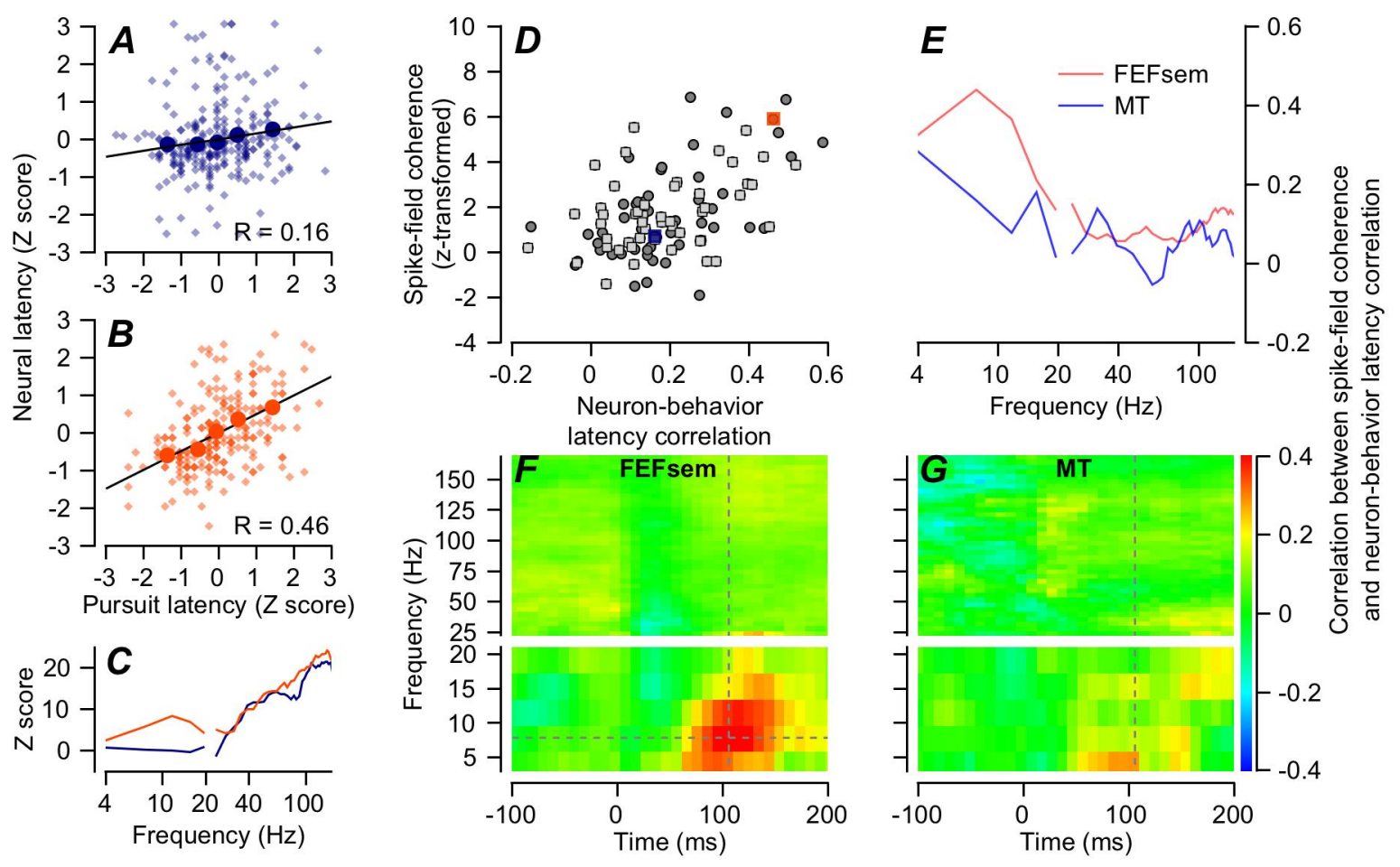

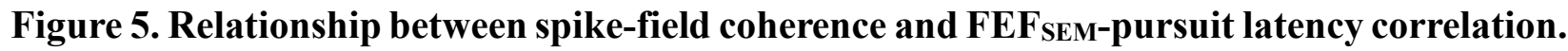
A-B: Plots of z-scored neural latency versus z-scored pursuit latency for 2 example neurons. Navy blue and orange circles show data for the 5 quintiles based on pursuit latency and smaller diamond symbols show measurements for individual trials. Lines were obtained by regression. FEF $_{\text {SEM}^{-}}$ pursuit latency correlation of the neurons in panels $\mathbf{A}$ and $\mathbf{B}$ are low versus high. C: Z-scored spikefield coherences of the two example neurons as a function of frequency measured at a time window centered at $106 \mathrm{~ms}$ from motion onset. Colors match A and B. D: Each symbol shows data for a different neuron and the scatter plot shows the correlation between z-scored spike-field coherences and FEFSEM-pursuit latency correlations. Orange and navy-blue squares are data of example neurons. Dark gray circles and light gray squares are data from monkeys Re and Xt. E: Red and blue lines show the correlation between z-scored spike-field coherences and neuron-behavior latency correlations measured at a time window centered at $106 \mathrm{~ms}$ averaged across all recording

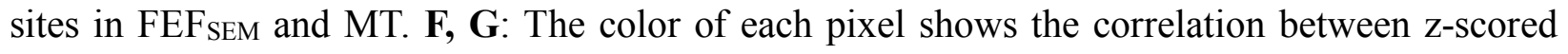
spike-field coherence and neuron-behavior latency correlations for example recording sites in $\mathrm{FEF}_{\text {SEM }}(\mathbf{F})$ and MT $(\mathbf{G})$, plotted as a function of frequency in the coherences and time from the onset of target motion. The intersection of the dashed lines in $\mathbf{F}$ shows the time and frequency used for panel D. 

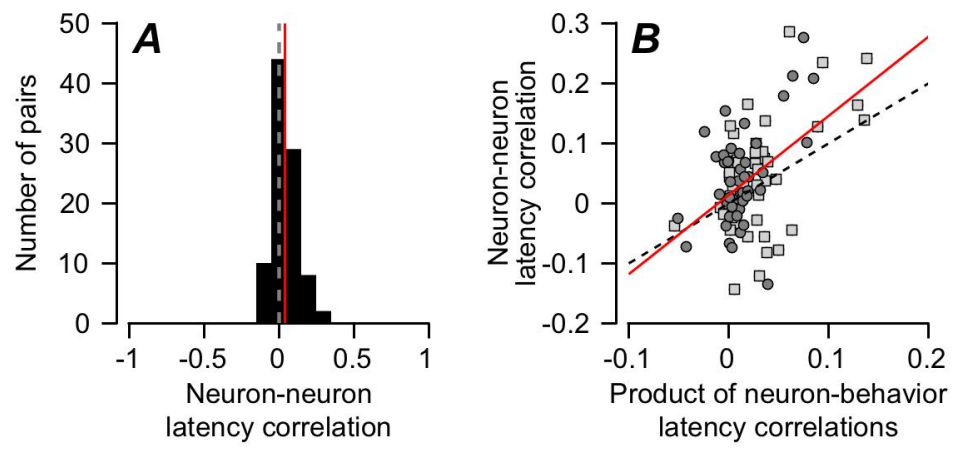

Figure 6. Neuron-neuron latency correlations in pairs of FEFSEM neurons. A: Distribution of neuron-neuron latency correlations between pairs of FEFSEM neurons. Vertical red line shows the mean correlation. B: Each point plots data for a different pair of FEF SEM neurons, and the scatter plot shows the relationship between the product of FEFSEM-pursuit latency correlations and neuron-neuron latency correlations. The dashed line has a slope of one and the red line is a regression fit. Dark gray circles and light gray squares are data from monkeys Re and Xt. 

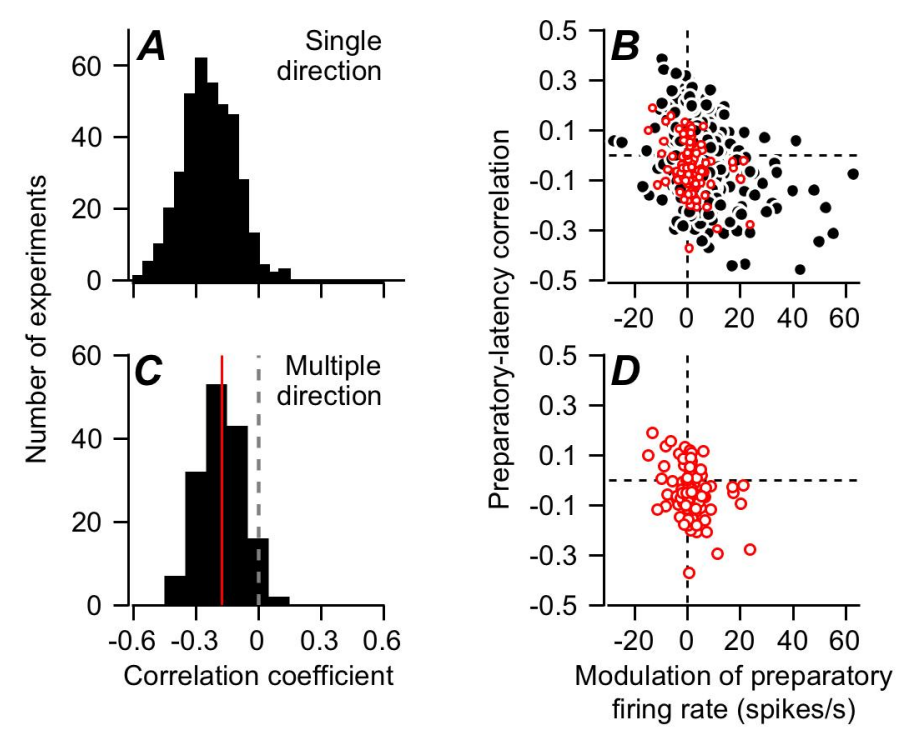

Figure 7: Relationship between preparatory activity and behavioral latency. A, C: Distributions of trial-by-trial correlations between duration of fixation and pursuit latency. A reanalyzes previously-reported single-direction experiments in monkeys $\mathrm{Xt}$ and $\mathrm{Re}$, while $\mathbf{C}$ reports results for the experiments in this paper, from the same 2 monkeys but for blocks of trials with 2 or 4 directions of randomly-interleaved target directions. B, D: Scatter plots showing the trial-by-trial correlation of preparatory activity at the end of fixation with pursuit latency versus the modulation of preparatory activity across fixation. Each symbol shows results for a different experiment. Again, B shows data for previous single-direction experiments and $\mathbf{D}$ shows data for multiple-direction experiments in the present paper. Red symbols in $\mathbf{D}$ have been replotted in $\mathbf{B}$. 

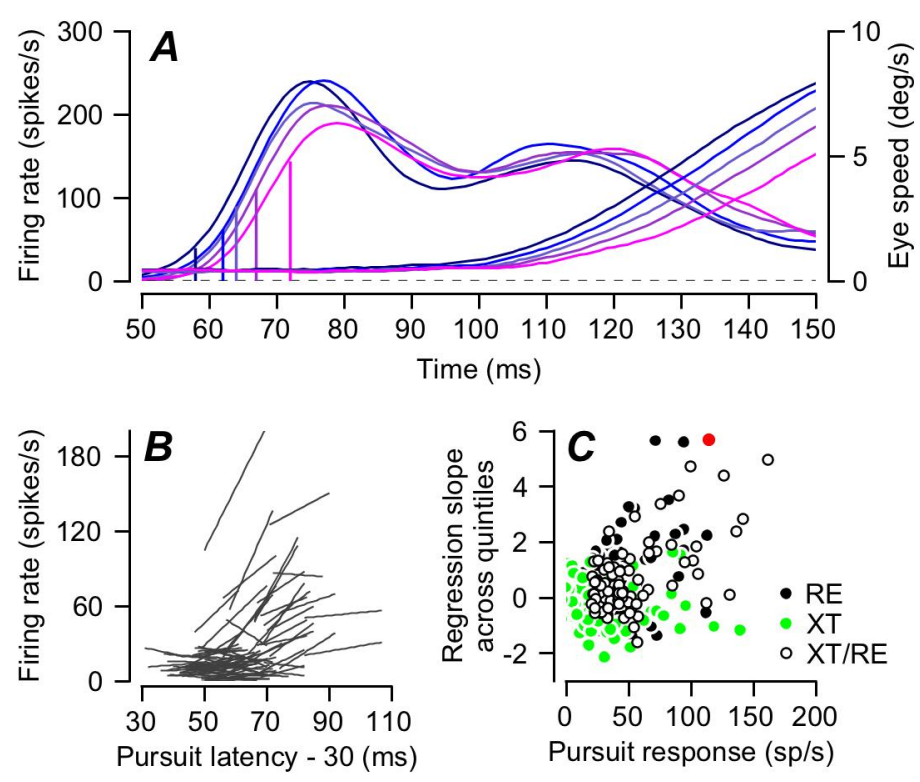

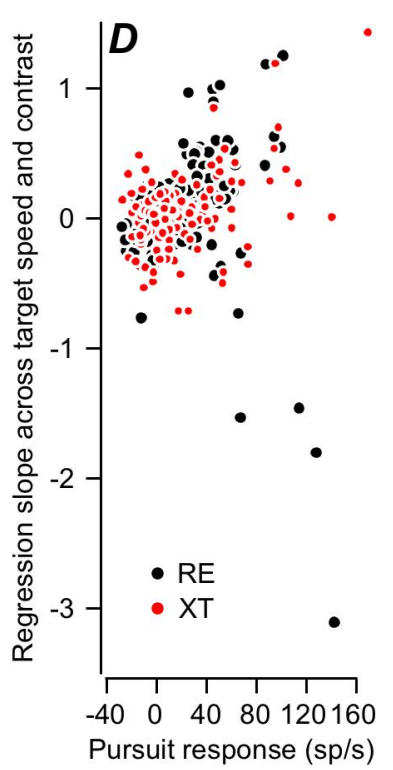

Figure 8. Direct tests of the ramp-to-threshold hypothesis for individual neurons in FEF SEM. A: Firing rate (left-hand traces) and eye velocity (right-hand traces) for an example neuron divided into quintiles according to pursuit latency in individual trials and plotted as a function of time from the onset of target motion. The 5 vertical lines on the firing rate traces show the values of firing rate $30 \mathrm{~ms}$ before the latency of eye velocity in each quintile. B: Linear regression lines fitted to each FEF for the 5 quintiles of pursuit latency. Each line shows data for a different neuron. C: Slope of the regression line versus amplitude of the pursuit-related response. A slope of zero would support the ramp-to-threshold hypothesis. Each symbol shows a different neuron. Open symbols show data from this paper, red symbol shows the neuron in $\mathbf{A}$, and filled green and black symbols show results of the same analysis for our earlier single-direction experiments on the same two monkeys. D: Same plot as $\mathbf{C}$, but now using data solely from our earlier single-direction experiments and plotting the regression slopes for points taken from targets of high- or low-contrast moving at 2 , 10 , or $20 \mathrm{deg} / \mathrm{s}$. 


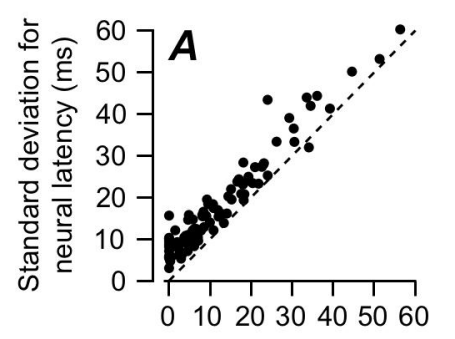

Standard deviation for latency of underlying spiking probability (ms)
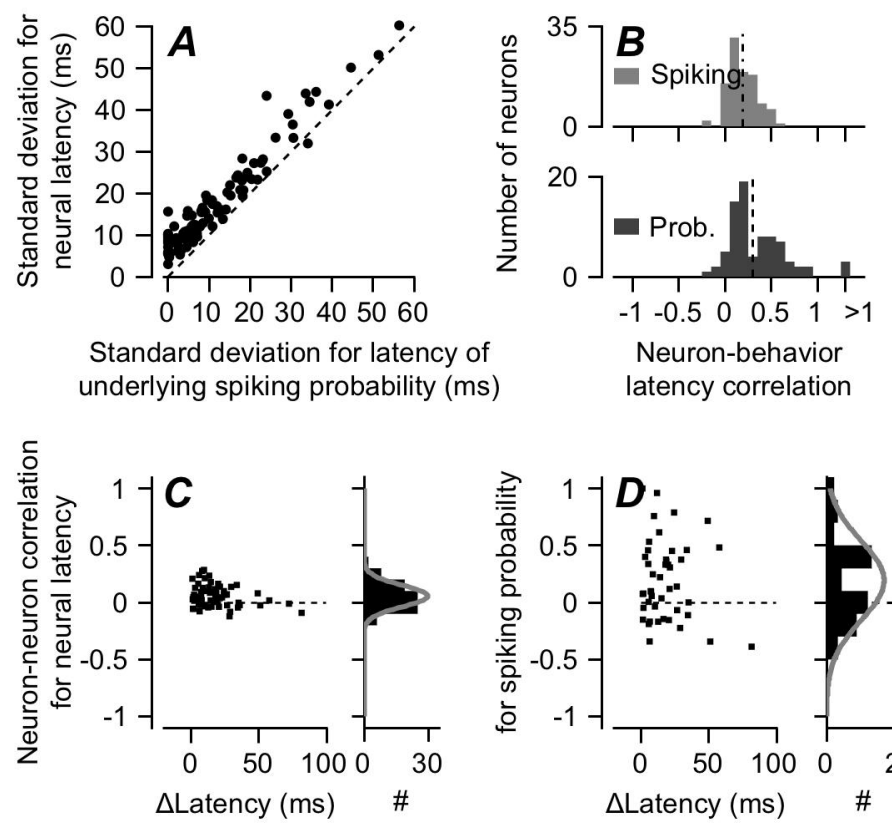

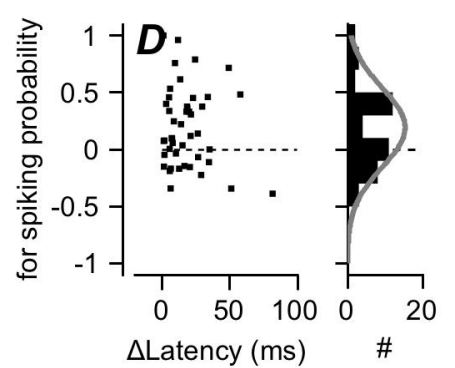

Figure 9. Computational analysis for the effect of neuron-neuron latency correlation on behavioral latency variation. A: Relationship between latency SD of firing rate and latency SD of underlying firing probability of spiking. Each symbol shows one neuron. Dashed line is the

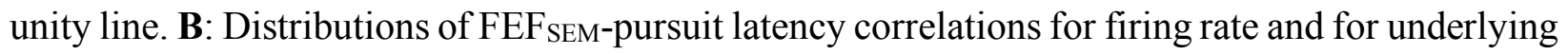
probability of spiking. The number of neurons included on this analysis is different from the previous figures. C: Distribution of neuron-neuron latency correlation for firing rate as a function of the mean latency differences between neurons. D: Distribution of neuron-neuron latency correlation for underlying probability of spiking in relation to mean neural latency differences. E: Predictions for pursuit behavior based on decoding a model $\mathrm{FEF}_{\mathrm{SEM}}$ population for different values of downstream noise. Black and gray filled areas show standard deviation of latency and neuronbehavior latency correlation averaged across 10 simulations. Vertical line shows SD of $3.5 \mathrm{~ms}$ for Gaussian noise added downstream. 